\title{
North-South Climate Change Negotiations: a Sequential Game with Asymmetric Information
}

\author{
A. Caparrós, J.-C. Péreau ${ }^{\dagger}$ and T. Tazdaï ${ }^{\ddagger}$ \\ Published in Public Choice, 2004, vol 121, issue 3-4, pp. \\ 455-480, december.
}

\begin{abstract}
This article determines the conditions under which the Southern countries should act together, or separately, while negotiating with the North about climate change policy and about the conditions for future Southern engagement. The paper models the international negotiations with complete and with asymmetric information in a dynamic framework. Results show that, depending on their characteristics, the different players can obtain benefits delaying the moment of the agreement.
\end{abstract}

JEL classification: C78, D74, H77.

Key words: Bargaining theory, Asymmetric information, Climate change, International cooperation.

*Department of Economics, Institute of Economics and Geography (IEG), Spanish Council for Scientific Research (CSIC), Pinar 25, 28006 Madrid, Spain. Email : acaparros@ieg.csic.es. Previously CNRS-EHESS-CIRED.

†OEP, University of Marne-la-Vallée, Cité Descartes, 5 bd Descartes, Champs sur Marne, Marne la Vallée Cedex 2, France. Email : pereau@univ-mlv.fr.

${ }^{\ddagger}$ CNRS-EHESS-CIRED Jardin Tropical, 45 bis av de la belle Gabrielle, 94736 Nogent sur Marne Cedex, France. Tel. +33.1.43.94.73.73. Fax. +33.1.43.94.73.70. Email : tazdait@centrecired.fr. (Corresponding author). 


\section{Introduction}

In 1992 virtually all the countries of the world signed, and ratified, the United Nations Framework Convention on Climate Change whose ultimate objective is to achieve the 'stabilization of greenhouse gas concentration in the atmosphere at a level that would prevent dangerous anthropogenic inference with the climate system'. Five years later, the Kyoto Protocol was signed, engaging the countries included in Annex I (OECD and economies in transition) to reduce their overall emission by five per cent in 2012 compared to 1990. This agreement was further discussed and refined, and in 2001, almost all the countries in the world (with the only significant absence of the United States of America) agreed on the Marrakech Accords, which are expected to enable enforcement of the Kyoto Protocol (this requires that countries accounting for $55 \%$ of Annex I carbon dioxide emissions ratify it ${ }^{1}$.

Although it is fair to acknowledge that the industrialized countries (IC) are the main historic, and present, causers of anthropogenic climate change, the solution of the problem will need the implication of the developing countries (DC) in a more comprehensive way than the one proposed in the Kyoto Protocol (based on projects). This is especially true if we assume that the $\mathrm{DC}$ will be able to develop as fast as they need. In fact, the incorporation of the DC is the main issue on the negotiation agenda and the absence of binding targets for the DC was one of the main arguments used by President Bush to reject the Kyoto Protocol.

In the next negotiation rounds we shall see the North (the Annex-I countries which have ratified the Kyoto Protocol) negotiating with the South (Non-Annex I countries) the most convenient way to bring them on board. The North, led by the European Union, has recognized its historical responsibility (in official texts but also accepting to be the first one to reduce) and should be ready to compensate the South in some way for accepting limitations to their urgently needed development. This compensation could take the form of monetary transfers but could also be based on other type of advantages.

Until now the DC have talked in the international climate change arena with a single voice, the so-called "G77 and China" group. Nevertheless, until now, the DC, although Parties of the negotiations, have not been affected by binding reductions. Thus, they have been less involved in the "hard part" of the negotiations. In the new context, the Southern countries have to decide whether they continue to talk with a single voice or whether they split up into two or more groups (the G77 and China is a highly heterogeneous group, including small countries highly vulnerable to climate change and countries like Saudi Arabia strongly interested in oil exports). Talking together gives bargaining power, but under some circumstances, it may be more convenient to split up to take advantage of internal differences. Rotillon et al. (1996) analysed this issue with complete information

\footnotetext{
${ }^{1}$ As well as 55 countries, including Annex I as well as Non-Annex I countries.
} 
and particular benefit functions. Nevertheless, official statistics in the South are rare and incomplete and real knowledge about the abatement costs and options in the South are not at all available to the North. On the contrary, due to the high amount of existing studies, and due to the implementation of the Kyoto Protocol, the South will have almost complete information about the cost and benefit functions of the North. Hence, we discuss this issue in the context of asymmetric information and with general functional forms.

Finally, if the Southern countries decide to split up into two groups (e.g.), these groups can be complementary or substitutable. We call them complementary if the North needs to sign an agreement with both groups to be effective. Substitutable implies that an agreement with one of the groups is enough to produce positive benefits. At first glance, the Southern groups should be considered substitutable in the case of climate change since if one group, e.g. representing $50 \%$ of the Southern emissions, accepts to reduce its future emissions (or to increase them less) this will have a positive effect on climate change. Nevertheless, the opposite situation is also possible. As described above, in 1997 the Kyoto Protocol was signed, but stating that it will only come into force if countries representing $55 \%$ of Annex I emissions ratify it. This gave Russia and other countries a lot of bargaining power since they became complementary, especially after the withdrawal of the United States. This situation might be repeated if an initial Protocol states that the $\mathrm{DC}$ will only be obliged to reduce their emission patterns if DC representing $55 \%$ (e.g.) of Non-Annex I emissions ratify it ${ }^{2}$. Hence, we discuss in our model the circumstances under which the South is interested in pushing for such a situation.

Another relevant characteristic of international negotiations, and in particular of climate change negotiations, is that they take a great deal of time to conclude. In principle, if negotiators were rational, we should expect them to finish in one single negotiation round. Nevertheless, the delay in the negotiations can be used, and has been used in the past, to take advantage and to gain better knowledge of the characteristics of the opponent. Therefore, we model the negotiations in a dynamic framework to analyse the circumstances under which the Parties benefit from delaying the agreement.

Game theory literature on cooperation in the context of climate change has followed two approaches ${ }^{3}$, although both of them assimilate cooperation and coordination of environmental policies. On the one hand, we have what Tulkens (1998) called the thesis of the "small stable coalitions" (Carraro and Siniscalco, 1993; Barret, 1994; Péreau and Tazdait, 2001; among others). On the other hand, we have the thesis of the "grand stable coalition" (Chander and Tulkens, 1997; Egteren and Tang, 1997; Funaki and Yamato, 1999; Helm, 2001; among others). According to the first approach, only a small number of countries will

\footnotetext{
${ }^{2}$ In fact, this kind of clause is common in international agreements.

${ }^{3}$ For a survey see Finus (2001).
} 
accept to sign an agreement (the stability concept used comes from the analysis of cartels in industrial economics). In this framework, a coalition including all countries is not sustainable since the benefits arising from cooperation are a public good (distributed to all countries), while the costs are borne only by the countries forming the coalition. On the contrary, in the second approach all countries are interested in cooperation (taken individually or in sub-groups). According to Chander and Tulkens $(1995,1997)$ an agreement should not only be based on pareto optimality to be effective, but should also prevent any subgroup of countries to be interested in secession. Hence, the objective is to set up a burden-sharing rule able to favour the cooperation of all. However, these two approaches have in common that the negotiating process has been completely ignored, as well as significant characteristics like information asymmetry, countries heterogeneity ${ }^{4}$, or even the possibilities of renegotiation. In the non-cooperative games evoked by Carraro and Siniscalco, negotiation is modelled as simultaneous discourse, not leaving any space to dialogue (i.e. the possibility of carrying out counter-propositions). In the models of endogenous coalition formation (Chander and Tulkens, 1995, 1997), cooperation is the result of the direct intervention of a third party: a supranational authority acting as a central planner. This central planner is assumed to be good-willed, to defend the global interests and to have enforcement power; somehow irrealistic assumptions in the context of real climate change negotiations.

To overcome these limitations, we analyse future climate change negotiations in a dynamic framework, with asymmetric information, with heterogeneous coalitions and explicitly including the possibility to make counter-propositions (remark that bargaining games are strictly competitive games, since this may be useful for the interpretation of our results). In technical terms, we develop a sequential game with asymmetric information in a bilateral, as well as in a multilateral framework. Therefore, we deal with hidden knowledge issues and signalling theory; and look for pooling and separating equilibria. This model is set out using general functional forms and could therefore be applied to most of the current and future discussions between the North and the South. It applies, more generally, to all circumstances where negotiations take place between, on the one hand, one player whose characteristics are well known and who is ready to pay the other players to obtain something, and, on the other hand, one or two players whose characteristics are not known.

The article is organised as follows. In section 2 we present the general model. In section 3, we analyse the outcomes of the game with complete information. The study of the negotiation with incomplete information is proposed in section 4. Finally, section 5 highlights the main conclusions.

\footnotetext{
${ }^{4}$ Most of the work with heterogeneous countries is based on numerical simulations (Barrett, 1997; Botteon and Carraro, 1997, 1998) and hence does not provide general results. The theoretical work available has been developed within the highly restricted framework of countries characterised by specific welfare functions (Hoel, 1992; Heal, 1994).
} 


\section{The model}

We analyse a bargaining game between the North (Annex I countries which have ratified the Kyoto Protocol), which is assumed to be neutral to risk, and a group of developing countries (e.g. the G77 and China). The negotiation is about the value of the transfers that the North $(\mathrm{N})$ will grant to countries of the South $(\mathrm{S})$ willing to cooperate and commit themselves. The Southern countries are assumed to be identical and $C$ in number. To assume identical countries could be seen as a limitation (see above); however, it is important to recall that we are assuming that all the countries of the Southern coalition talk with a single voice. Therefore, there is no problem in considering that a country like China has more votes (parts of $C$ ) in the internal vote (in fact, $C$ could also refer to the amount of emissions as in the example described in the introduction). We call $0<\delta_{i}<1$ the discount rate of agent $i, i=N, S$. The monetary transfer (or other type of advantage) granted by the North is noted $\tau$. The benefit for the North is the difference between the abatement reached $(B(C)=B)$, and the cost of the transfers to the $C$ countries of the Southern coalition $(\tau C)$ :

$$
\Pi(\tau, t)=\delta_{N}^{t}[B-\tau C]
$$

The utility function of the Southern coalition is:

$$
U(\tau, t)=\delta_{S}^{t}[(\tau-b) C]
$$

We assume that the minimum amount of transfers asked for by the Southern coalition $(b)$ is decided in an internal vote among the coalition (as it occurs in the international negotiations, where G77 and China have private meetings where they decide their position). With this amount of transfers $b$ the utility is zero. We also define the "reservation transfer", as the maximum transfer that the North can accept. This amount is given by the benefits that the North obtains from the reductions in the South (we assume that the negotiation is about the amount of transfers for a given level of Southern effort). The maximum transfer level is $B / C$, and the benefit for the North would be zero. That is, the negotiation area is bounded by $b$ and $B / C$. Thus, for $B / C<b$, the agreement set is empty. To make the agreement possible, we assume $B>C b$. All the elements of the negotiation are known, except the minimum amount of transfers that is acceptable to the Southern group. This amount can have two possible values $b^{-}$and $b^{+}$(i.e. $b \in\left\{b^{-}, b^{+}\right\}$), where $b^{-}<b^{+}<B / C$. The Northern negotiator has an a priori probability function for these values: $p_{N}\left(b^{+}\right)=p^{+}$and $p_{N}\left(b^{-}\right)=p^{-}=1-p^{+}$.

The players propose offers alternatively, which refer to the amount of transfers for a given effort. The negotiation goes on with no time limit. We call the game where the North (respectively the South) makes the first offer $J_{N}^{\infty}\left(J_{S}^{\infty}\right)$. Let us analyse the structure of the $J_{N}^{\infty}$ game. 
The North proposes an amount of transfers $\tau_{N}$. The South accepts (Yes) or refuses (No) the proposition. If the South accepts, an agreement is signed in the first period. The objective functions of the agents are:

$$
\Pi\left(\tau_{N}, 0\right)=B-\tau_{N} C \text { and } U\left(\tau_{N}, 0\right)=\left(\tau_{N}-b\right) C
$$

If the South rejects the offer, the negotiation continues with a counter-offer from the South. If the North accepts this new offer, an agreement is signed in the second period, with an amount of transfers $\tau_{S}$. Thus:

$$
\Pi\left(\tau_{S}, 1\right)=\delta_{N}\left[B-\tau_{S} C\right] \text { and } U\left(\tau_{S}, 1\right)=\delta_{S}\left[\left(\tau_{S}-b\right) C\right]
$$

Otherwise, the negotiation starts again with a Northern offer $\tau_{N}$. In the case of an agreement:

$$
\Pi\left(\tau_{N}, 2\right)=\delta_{N}^{2}\left[B-\tau_{N} C\right] \text { and } U\left(\tau_{N}, 2\right)=\delta_{S}^{2}\left[\left(\tau_{N}-b\right) C\right]
$$

And so on. In the case of perpetual disagreement we get :

$$
\Pi\left(\tau_{0}, \infty\right)<\Pi(\tau, t) \text { and } U\left(\tau_{0}, \infty\right)<U(\tau, t) \text { for the final } t .
$$

We assume that both agents have a given preference for the present. Hence, for two moments in time $t$ and $s$, with $t<s$, we have:

$$
\Pi(\tau, t) \geq \Pi(\tau, s) \text { and } U(\tau, t) \geq U(\tau, s)
$$

with $\tau=b^{-}, b^{+}$. The inequality will be strict if $\Pi>0$ (respectively $U>0$ ).

Since the acceptation of an offer closes the negotiation, a history in time $t, h^{t}$, describes a possible evolution of the negotiations from the beginning at date 0 until date $t$ (including the behaviour in $t$ ). More precisely, it gives the sequence of propositions leading to a disagreement, if there were propositions, knowing that the North revises its beliefs taking into account the history. Call $H$ the set of all the possible histories and $H^{t}$ the set of all the possible histories with a duration $t$. A pure strategy for $i$ is a function $f_{i}$ where:

$$
f_{i}^{t}: H_{i}^{t-1} \rightarrow\left[b^{-}, B / C\right] \cup\{\text { Yes, No }\}
$$

specifies after each history $h_{i}^{t-1}\left(\in H_{i}^{t-1}\right)$ the behaviour of $i$ at time $t$. In particular, it tells us if after $h_{i}^{t-1}, i$ will propose a new offer, or refuse or accept the offer of $j$. Hence, a history is formed either by a proposition between $b^{-}$ and $B / C$, or by a refusal or an acceptation. This last choice can only appear at $t$. The result of the negotiation will also depend upon the beliefs of the North. The resolution of the dynamic game with incomplete information is based on the concept of sequential equilibrium (Kreps and Wilson, 1982). By definition, a set of strategies and a family of beliefs form a sequential equilibrium if the following 
two conditions hold: (i) the strategies of both players are optimal in all the points of the game, given the beliefs of the North, and (ii) the beliefs of the North are Bayesian $^{5}$.

\section{Complete information}

\subsection{Bilateral negotiation}

Following a similar resolution method to the one proposed by Rubinstein (1982), we calculate the unique perfect equilibrium with complete information to which both players agree immediately in the first negotiation round (see Hovi (2001) for an alternative application of this methodology to the Clean Development Mechanism). The minimum amount of transfers $b$ requested by $S$ is supposed to be common knowledge. Let $\tau^{*}(N, S)$ be the equilibrium transfer when the North opens the negotiations and $\tau^{*}(S, N)$ the equilibrium transfer when it was proposed by the South ${ }^{6}$. We obtain:

$$
\tau^{*}(N, S)=\frac{b C\left(1-\delta_{S}\right)+\delta_{S}\left(1-\delta_{N}\right) B}{\left(1-\delta_{N} \delta_{S}\right) C}
$$

and

$$
\tau^{*}(S, N)=\frac{B\left(1-\delta_{N}\right)+b C \delta_{N}\left(1-\delta_{S}\right)}{\left(1-\delta_{N} \delta_{S}\right) C}
$$

Concerning the position of the players in the game, we can formulate the following corollary. It says that $\mathrm{N}$ (respectively $\mathrm{S}$ ) prefers to play the game when it opens the negotiations $\left(J_{N}^{\infty}\right.$, respectively $\left.J_{S}^{\infty}\right)$, instead of the opposite when it responds $\left(J_{S}^{\infty}\right.$, respectively $\left.J_{N}^{\infty}\right)$.

Corollary 1 If $J_{N}^{\infty}$ and $J_{S}^{\infty}$ are two games with perfect information, being $b$ common knowledge, we obtain the following two expressions:

$$
\begin{aligned}
& J_{N}^{\infty} \succ_{N} J_{S}^{\infty} \\
& J_{S}^{\infty} \succ_{S} J_{N}^{\infty}
\end{aligned}
$$

Proof. We can see that:

$$
\tau^{*}(S, N)>\tau^{*}(N, S) \Longleftrightarrow B>C b
$$

Since by definition $B>C b$, the inequality always holds.

$\mathrm{S}$ wishes to obtain the highest amount of transfers and prefers the situation where

\footnotetext{
${ }^{5}$ We could also adopt a perfect bayesian equilibrium concept. However, as Kreps and Wilson (1982) show, this concept has the drawback that it defines a great amount of solutions, while the sequential equilibrium permits to restrain them.

${ }^{6}$ These transfer equilibria are derived from a backward induction reasoning (Rubinstein, 1982).
} 
$\tau=\tau^{*}(S, N)$, i.e. the amount of transfers that it obtains in the game where it talks first. On the contrary, $\mathrm{N}$ wishes to obtain the lowest amount of transfers and prefers the situation where $\tau=\tau^{*}(N, S)$, i.e. the amount of transfers that it obtains in the game where it talks first.

\subsection{Multilateral negotiation}

Let us now consider the case where the South is organised in two separate groups, $S_{i}, i=1,2$, representing $C_{i}$ countries, with $C_{1}+C_{2}=C$. The benefit obtained by the North from the abatement of group $i$ is noted $B_{i}, i=1,2$. The aim is to study the union and disunity behaviours of the groups in complete information according to their degree of complementarity and substitutability. We assume that within each group, the members vote to determine their minimum level of transfer claims. Let $b_{i}(i=1,2)$ be the transfer requested by the group $i$ (resulting from the internal vote within each group) and $b$ the transfer requested by the groups while acting together (resulting from the internal vote within the group formed by both sub-groups).

The bargaining process has the same characteristics as previously described. The game consists in alternative offers and the North makes the initial ones (one offer to each group). If one group accepts the offer while the other refuses, the negotiation with the former group is finished; going on with the latter group. If both groups simultaneously refuse (accept), the negotiation keeps going on (ends) with both groups. This process goes on until an agreement is reached.

Let $0<\delta_{S}<1$ be the fixed discount rate of the groups (joined or separated). We have $\forall i=1,2$ :

$$
\tau^{*}\left(N, S_{i}\right)=\frac{b_{i} C_{i}\left(1-\delta_{S}\right)+\delta_{S}\left(1-\delta_{N}\right) B_{i}}{\left(1-\delta_{N} \delta_{S}\right) C_{i}}
$$

Both groups will accept to form a unique alliance if, by their cohesion, they reach a more satisfactory level than they would have reached acting separately:

$$
U_{1}+U_{2}<U
$$

where $U_{i}$ is the utility of the groups $(\forall i=1,2)$. Condition (6) involves:

$$
b C-b_{1} C_{1}-b_{2} C_{2}<B-B_{1}-B_{2}
$$

Let us define $V_{i}=(B-b C)-\left(B_{j}-b_{j} C_{j}\right), i \neq j$, the maximum contribution of group $i$ to the benefit of the North. Condition (7) is rewritten as follows:

$$
V_{1}+V_{2}>B-b C
$$

The two Southern groups should act together when the North benefits from negotiating separately with each of them. This only happens if the distribution rule 
among the members of the Southern group allows them to obtain more together than what they would obtain separately. The North is only motivated to favour two bilateral negotiations if it intends to propose a low level of transfers. In this configuration, by standing separately, the bargaining power of the South is lower, as their threat to refuse any agreement is less credible. If one group tries to use that threat, the North will propose a slightly more interesting offer to the other group. Being confronted with two distinct groups, it could propose a low level of transfers and take advantage of the disunity and lead both Southern groups to accept its offer. To foil that strategy, the two groups have to act together.

If condition (8) is not respected, the Southern groups should participate separately in the negotiations. In this case, the North is inclined to propose a high level of transfers from the beginning of the negotiation. Since the North proposes a high level of transfers, its intention is to conclude an agreement as soon as possible. Each group is therefore incited to take advantage of that situation by accepting the overbid game. The separation of the groups accentuates this behaviour and increases their bargaining power. Hence, we obtain the following proposition:

\section{Proposition 1}

1. When $V_{1}+V_{2}>B-b C$, a unique subgame perfect equilibrium exists in which the groups act jointly.

2. When $V_{1}+V_{2}<B-b C$, a unique subgame perfect equilibrium exists in which the groups act separately.

The outcome of the negotiations depends widely on the degree of commitment of the North. It defines the whole process of negotiations by making the first offer. If the Southern countries anticipate a moderate commitment, they will be united during the negotiations, since that will give them significant bargaining power. Conversely, if they anticipate a high commitment, they will be dissociated into two separate groups.

We will now analyse the behaviour of the Southern groups according to their degree of substitutability and complementarity. The groups are said complementary when the two groups have to sign the agreement so that benefits can arise (see introduction). They are called substitutable when the agreement can be reached with only one of the groups. If we accept perfect complementarity, an agreement signed by only one group has no impact. Therefore, $\forall C_{i}, i=1,2$, we have $V_{1}=V_{2}=0$. Hence:

$$
V_{1}+V_{2}<B-b C
$$

Since relation (9) always holds for the complementary groups, we assume that they will act separately during negotiations. In this way both can unilaterally compromise the negotiations in case of disagreement. On the other hand, when 
the groups are substitutable, they had better act together to preserve their bargaining power. The following expression is found inverting equation (6):

$$
V_{1}+V_{2}>B-b C
$$

According to the North, the inequality (10) means that the maximum additional contribution to the Northern payoff of the two united groups is low. Thus, an agreement with one of the groups is enough. We obtain the following proposition:

\section{Proposition 2}

1. When the two groups are perfectly complementary, they will act separately.

2. When the two groups are perfectly substitutable, they will act jointly.

When the Southern groups are complementary; the North would like to negotiate with the united groups. Conversely, the Southern groups will adopt the asymmetric behaviour. Being complementary, they are aware of being the driving forces and that incites them to become two distinct groups. Such behaviour gives them bargaining power and allows them to request a higher amount of transfers.

When the groups are substitutable; the North needs to conclude an agreement with only one of them. Conversely, the Southern groups are incited to act united to preserve their bargaining power. Nevertheless, that alliance is fragile since one of the groups can betray its partner accepting a slightly lower level of transfers, which guarantees the signing of an agreement.

\section{One-sided incomplete information}

\subsection{Bilateral negotiation}

We now analyse the game in which the North opens the negotiations and does not know the amount of transfers requested by the Southern countries. We might justify this situation as follows: the North has complete, easily accessible statistics, as well as a high amount of studies estimating the costs of climate change policy in the North. In addition, the North (formed by the IC which have ratified the Kyoto Protocol) will have started its climate change policy and revealed its reduction potentials. On the contrary, in most of the Southern countries statistics are incomplete and the knowledge about the cost of alternative climate change policies is not well known. Therefore, the South has better information about the amount of transfers that it can request compared to the information available to the North about the minimum level of transfers (or other benefits) that the South would accept. 
To simplify, we assume that the amount of transfers requested by the Southern group can take two values: $b^{-}$and $b^{+}$, with $b^{-}<b^{+}<B / C$. Either it is low, and we will call the group type $b^{-}$, or it is high and the group will be of type $b^{+}$. According to Harsanyi (1967), this game is formalised by introducing a new player, Nature, which selects the group $b^{+}$(respectively $b^{-}$) as the Northern opponent with probability $p^{+}$(respectively $1-p^{+}$). The distribution of probability is common knowledge.

Since we use the concept of sequential equilibrium, we have to specify the form of the beliefs of the North. We will define pessimistic beliefs ${ }^{7}$ (Rubinstein, 1988). Hence, each time an event of probability zero appears, the North considers that it is facing $b^{+}$.

The relation $b^{-}<b^{+}<B / C$ means that the North is in a position of strength faced with $b^{-}$, while it is in a position of weakness faced with $b^{+}$(who has stronger transfer demands). Within the game in complete information, the equilibrium transfer issued from the negotiation with $b^{+}$is higher or equal to the equilibrium transfer in the negotiation with $b^{-}$. Hence:

$$
\tau^{*}\left(N, S^{+}\right) \geq \tau^{*}\left(N, S^{-}\right)
$$

With one-sided incomplete information, $b^{-}$is interested in persuading the North that it has strong demands. Therefore, its strategy consists in adopting behaviour similar to $b^{+}$. Lemmas (1) to (4) show this result.

Lemma 1 If in any sequential equilibrium of the game $J_{N}^{\infty}$, two types of Southern groups propose a counter-offer, they will make the same one. In this case, $b^{-}$is interested in suggesting a counter-offer identical to the one proposed by $b^{+}$.

Lemma 2 In any sequential equilibrium of $J_{N}^{\infty}$ :

a) If $b^{-}$refuses a proposition $\tau_{N}, b^{+}$is interested in doing as well.

b) Conversely, if $b^{+}$accepts a proposition $\tau_{N}$, it is in the interest of $b^{-}$to do as well.

Lemma 3 In any sequential equilibrium of $J_{N}^{\infty}$, a group, whatever its type, will accept any offer $\tau_{N}$ that satisfies:

$$
\tau_{N} \geq\left(1-\delta_{S}\right) b+\delta_{S} \tau^{*}\left(S^{+}, N\right)
$$

As the range of possibilities of $b^{-}$is larger than those of $b^{+}$, there are levels of transfer $\tau_{N}$ likely to be accepted by the first and refused by the second. The following lemma (4) confirms that.

\footnotetext{
${ }^{7}$ We retain that type of beliefs among the family of possible beliefs (optimistic, passive or continued0x2026) since they are the most economically relevant within this model.
} 
Lemma 4 In any sequential equilibrium of $J_{N}^{\infty}$, if:

$$
\left(\tau^{*}\left(S^{+}, N\right)-b^{-}\right) \delta_{S}+b^{-} \leq \tau_{N}
$$

$b^{-}$accepts the proposition $\tau_{N}$ while $b^{+}$rejects it and offers $\tau^{*}\left(S^{+}, N\right)$, which will be accepted by $N$.

Lemmas (1) to (4) lead us to propositions (3) to (5) for the one-sided incomplete information game.

Proposition 3 In any sequential equilibrium of the game $J_{N}^{\infty}$ where the North is characterised by pessimistic beliefs and where equation (13) is satisfied, we obtain the following results:

1. If:

$$
p^{+}<\frac{C\left[\tau^{*}\left(N, S^{+}\right)-\tau_{N}\right]}{B\left(1-\delta_{N}\right)+C\left[\delta_{N} \tau^{*}\left(S^{+}, N\right)-\tau_{N}\right]}=g
$$

The North proposes the level of transfers $\tau_{N} . b^{-}$accepts it while $b^{+}$refuses it to make a counter-offer $\tau^{*}\left(S^{+}, N\right)$, accepted by the North.

2. If $p^{+}>g$, the North proposes the level of transfers $\tau^{*}\left(N, S^{+}\right)$which is immediately accepted by the Southern groups whatever their type.

3. If $p^{+}=g$, two cases are conceivable:

- Either the North proposes the transfer $\tau^{*}\left(N, S^{+}\right)$which is immediately accepted by the Southern group, whatever its type.

-Or it proposes the transfer $\tau_{N}$ that satisfies (13), but it is refused by $b^{+}$. The counter-offer of $b^{+}$is then equal to $\tau^{*}\left(S^{+}, N\right)$ and is accepted by the North.

The results of this proposition show that the asymmetry of information totally favours $b^{-}$as it is incited to adopt opportunistic behaviour. The North can however determine the real characteristic of its opponent. For that, it just has to propose a transfer $\tau_{N}$ that satisfies (13), which will be a sufficient incentive for $b^{-}$to accept and give up the option of making a counter-offer. Nevertheless, since $\tau_{N}>\tau^{*}\left(N, S^{-}\right), b^{-}$, although forced to reveal its characteristics, benefits from its opportunism. Let us add that the situation of the North is even more unfavourable if equation (13) does not hold. The following proposition illustrates this situation.

Proposition 4 In any sequential equilibrium of the game $J_{N}^{\infty}$ where the North is characterised by pessimistic beliefs and where the relation (13) does not hold, if the North opens the negotiations proposing $\tau^{*}\left(N, S^{-}\right)$, it will be refused by the Southern groups whatever their type. 
However, the asymmetry of information does not have a great impact on the date of signing of an agreement. The following proposition shows that an agreement will be rapidly obtained.

Proposition 5 In any sequential equilibrium of the game $J_{N}^{\infty}$ characterised by an asymmetry favourable to the Southern groups, the negotiations will never last more than two periods.

This proposition is similar to the one developed by Osborne and Rubinstein (1990). The idea is as follows: Suppose an agreement is reached at the period $n\left(n \in \aleph^{*}\right)$ on a proposition of the Southern group. Lemma (1) defines the behaviour of $b^{-}$. Its strategy consists in imitating the behaviour of $b^{+}$by making the same counter-offer. Thus, whatever the type of group, the counter-offers will be $\tau^{*}\left(S^{+}, N\right)$. In addition, the North is not interested in prolonging the negotiations until the period $n$, since it can accept the transfer $\tau^{*}\left(S^{+}, N\right)$ at the second period, obtaining higher earnings due to its preference for the present. Hence, if a proposition $\tau_{N}$ is refused by both types of Southern groups, the agreement can only emerge at the following period.

On the contrary, if the offer of first period $\tau_{N}$ is accepted by $b^{-}$, the North can determine the real characteristics of its opponent. Therefore the North knows that a counter-offer $\tau_{S}$ can only come from a $b^{+}$. Since it cannot increase its gain by refusing, the North will accept $\tau_{S}$. Thus, the negotiation will end at the second period.

Finally, if the offer $\tau_{N}$ is a sufficient incentive to be accepted by the two types of union, i.e. $\tau_{N} \geq \tau^{*}\left(N, S^{+}\right)$, the agreement will take place at the first period.

However, remark that this result is only valid for a situation where the incomplete information is reduced to two possibilities. If the problem gets more complicated, the agreement will no longer be reached at the second period.

Propositions (3) and (4) show that with asymmetry of information, two categories of agreements are likely to be signed. We can associate a sequential equilibrium to each of these types of agreements.

On the one hand, an agreement is possible where $b^{+}$accepts the equilibrium transfer obtained from the game without asymmetry of information. The equilibrium transfer is $\tau^{*}\left(S^{+}, N\right)$ and $b^{-}$accepts the offer $\tau_{N}$ defined by (13). We call this sequential equilibrium 'separating'. The North is certain to determine the characteristic of the Southern group. By proposing the transfer $\tau_{N}$, only $b^{-}$will accept it. In case of rejection, the North will suppose that its opponent is $b^{+}$.

On the other hand, an agreement concluded on a transfer corresponding to $b^{+}$, is highly profitable for $b^{-}$. This latter group obtains more than what it expected in complete information. We find here a 'pooling' sequential equilibrium. The agreement doesn't reflect the type of the contracting parties.

The importance of this second case depends strongly on the beliefs of the North. There is a monotonous relation between the value $p^{+}$and the possible 
emergence of a pooling sequential equilibrium. The higher (lower) the initial belief $p^{+}$of the North, the higher (lower) are the chances to meet an agreement with a transfer $\tau^{*}\left(N, S^{+}\right)$from the first negotiation round. Therefore, if the Southern group was able to emit in a pre-game phase a signal not corresponding to its type, the asymmetry could quickly favour them. Keeping personal information plays an essential role in the bargaining process.

Proposition (5) shows that time takes a new strategic dimension in the presence of asymmetry of information. In this case, time favours the Southern groups. We have seen that the strategy of $b^{-}$is to adopt opportunistic behaviour, by posting demands identical to the ones requested by $b^{+}$. This strategy allows it to emit a signal not corresponding to its own type. Furthermore, even if the negotiations continue, the North is not able to discover the real characteristic of the Southern group. Hence, to maintain the advantage granted by the asymmetry of information, $b^{-}$has to persevere and not change its strategy. Therefore, the North is relunctant to prolong the negotiations, since it will not be able to discover the real characteristics of its opponent. Taking into account the temporal dimension and its asymmetry of information, the North is eager to conclude an agreement quickly. Nevertheless, this configuration favours $b^{-}$. If the initial offer of the North is judged unsatisfactory, it will make a positive profit, equal to $\tau^{*}\left(S^{+}, N\right)-\tau_{N}$, since its counter-offer is higher than the one it would have requested in the absence of asymmetry of information. On the contrary, $b^{+}$ will receive a transfer corresponding to its characteristics (whatever the initial situation).

This proposition also shows that asymmetry of information could prolong the negotiations, although an agreement is likely to be achieved at the second period. This delay is a consequence of asymmetry of information, and corresponds to the time needed by the North to determine the Southern group's type.

\subsection{Multilateral negotiation}

We now analyse a negotiation between the North and two distinct Southern groups $S_{1}$ and $S_{2}$. When the North is confronted with the group $S_{i}(i=1,2)$ the associated distribution of probability is written $P_{N_{i}}\left(b^{+}\right)=p_{i}^{+}$and $P_{N_{i}}\left(b^{-}\right)=$ $1-p_{i}^{+}$. In extension to proposition (3), we have:

$$
g_{i}=\frac{C_{i}\left[\tau^{*}\left(N, S_{i}^{+}\right)-\tau_{N i}\right]}{B_{i}\left(1-\delta_{N}\right)+C_{i}\left[\delta_{N} \tau^{*}\left(S_{i}^{+}, N\right)-\tau^{*}\left(N, S_{i}^{-}\right)\right]} \quad i=1,2
$$

Since we focus on configurations involving a delay during the negotiation, we exclude the case where $p_{i}^{+}>g_{i},(i=1,2)$. When $p_{i}^{+}<g_{i}(i=1,2)$ three cases are conceivable according to the Southern groups' characteristics.

In the first configuration the group $j$ is of type $b_{j}^{-}$, and the group $k$ is of type $b_{k}^{+}, j \neq k$. Two sub-cases have to be considered. If both groups act together, 
they can claim either a transfer $b^{-}$, and obtain an agreement during the first stage, or a transfer $b^{+}$, which will extend the negotiation to the second period.

Sub-case 1. If acting together $b_{j}^{-}$and $b_{k}^{+}$request $b^{-}$, they are interested in separating themselves if and only if:

$$
U_{j}\left(\tau_{N j}, 0\right)+U_{k}\left(\tau^{*}\left(S_{k}^{+}, N\right), 1\right)>U\left(\tau_{N}, 0\right)
$$

That involves:

$$
\begin{aligned}
b_{j}^{-} & <\frac{\left(1-\delta_{N} \delta_{S}\right)\left(C b^{-}-C_{k} b_{k}^{+}\right)-\delta_{N}\left(1-\delta_{S}\right)\left(C b^{+}-C_{j} b_{j}^{+}-C_{k} b_{k}^{+}\right)}{\left(1-\delta_{N} \delta_{S}\right) C_{j}} \\
& -\frac{\left(1-\delta_{N}\right)\left(B-B_{j}-B_{k}\right)}{\left(1-\delta_{N} \delta_{S}\right) C_{j}}=\alpha_{1}
\end{aligned}
$$

Sub-case 2. If acting together $b_{j}^{-}$and $b_{k}^{+}$request $b^{+}$, they are interested in separating themselves if and only if:

$$
U_{j}\left(\tau_{N j}, 0\right)+U_{k}\left(\tau^{*}\left(S_{k}^{+}, N\right), 1\right)>U\left(\tau^{*}\left(S^{+}, N\right), 1\right)
$$

Solving:

$$
\begin{aligned}
b_{j}^{-} & <\frac{\left(1-\delta_{N} \delta_{S}\right)\left(C b^{+}-\delta_{S} C_{k} b_{k}^{+}\right)-\left(1-\delta_{S}\right)\left(C b^{+}-\delta_{N} \delta_{S} C_{j} b_{j}^{+}-\delta_{N} \delta_{S} C_{k} b_{k}^{+}\right)}{\delta_{S}\left(1-\delta_{N} \delta_{S}\right) C_{j}} \\
& -\frac{\left(1-\delta_{N}\right)\left(B-B_{j}-B_{k}\right)}{\left(1-\delta_{N} \delta_{S}\right) C_{j}}=\alpha_{2}
\end{aligned}
$$

Since it can easily be shown that $\alpha_{1}<\alpha_{2}$, for $b_{j}^{-}<\alpha_{1}$ whatever the claim of the union, the Southern groups favour disunity. For sub-case $1, b_{k}^{+}$is confronted with a delay while the union would lead to an agreement from the first stage. That delay represents the necessary time for $b_{k}^{+}$to separate itself from $b_{j}^{-}$and reveal its demands to the North. For the latter, these demands reveal the characteristics of $b_{k}^{+}$only if it has previously determined the type of $b_{j}^{-} \cdot b_{k}^{+}$would prefer disunity if it would obtain a higher benefit by showing its type. That requires that $b_{j}^{-}$ concludes an agreement on a low level of transfers in the first period.

For sub-case 2, the negotiation delay is less costly for $b_{k}^{+}$than in the previous case, since the delay would also occur acting together. Two simultaneous interpretations of the delay are possible. In the case of united groups, the delay represents the time needed by the North to infer the type of coalition it is confronted by. For the case of disunity, it describes the necessary time for $b_{k}^{+}$ to be dissociated from $b_{j}^{-}$. The sub-cases (1) and (2) correspond to a situation where the groups are complementary. This configuration reinforces the bargaining power of $b_{k}^{+}$. When it realizes that $b_{j}^{-}$wishes to demand a low transfer, it is 
interested in extending the negotiation to obtain a higher level of transfers in the second period.

When the inequalities (16) and (18) are inverted, we end up with a situation where the Southern groups are substitutable and act together. For the symmetric sub-case (1), despite union, the negotiation goes on. The common action does not give a sufficiently dissuasive bargaining power to end the negotiation in the first round. The bargaining power of the union is compensated by the presence of asymmetry of information, since the North uses the delay to determine the characteristics of the Southern group. Although the agreement is signed with the transfer $\tau^{*}\left(S^{+}, N\right)$, the union is confronted with a negotiation cost.

In the second configuration, we assume that the Southern group $j$ is of type $b_{j}^{+}$and the group $k$ of type $b_{k}^{+}$. In this case the negotiation will end in the second period even if there is no union. Disunity is favourable to the Southern groups if:

$$
U_{j}\left(\tau^{*}\left(S_{j}^{+}, N\right), 1\right)+U_{k}\left(\tau^{*}\left(S_{k}^{+}, N\right), 1\right)>U\left(\tau^{*}\left(S^{+}, N\right), 1\right)
$$

Solving:

$$
b_{k}^{+}<\frac{B_{j}+B_{k}-B+C b^{+}-C_{j} b_{j}^{+}}{C_{k}}
$$

Equation (20) corresponds to the condition of complementarity given by proposition (1). It shows that each group is interested in acting separately when their respective demands, although high, are not enough to take a relevant part of the benefit. Despite their complementarity, each group has room to manoeuver until a certain level. If this level is exceeded, the situation leads the groups to unite to obtain the bargaining power allowing them to reach an agreement based on a high level of transfers. This situation is associated to substitutability between the groups. In both cases, we find the initial interpretation of the negotiation delay. The delay becomes profitable to the North, it helps it to infer the characteristics of each group or the union of groups.

Finally, in the third configuration, we suppose that the group $j$ is of type $b_{j}^{-}$ and the group $k$ of type $b_{k}^{-}$. The delay can take place when the groups act jointly and require $b^{+}$. Disunity is profitable if:

$$
U_{j}\left(\tau_{N j}, 0\right)+U_{k}\left(\tau_{N k}, 0\right)>U\left(\tau^{*}\left(S^{+}, N\right), 1\right)
$$

Solving:

$$
\begin{aligned}
b_{k}^{-} & <\frac{\left(1-\delta_{N} \delta_{S}\right)\left(C b^{+}-C_{j} b_{j}^{-}\right)-\delta_{N}\left(1-\delta_{S}\right)\left(C b^{+}-C_{j} b_{j}^{+}-C_{k} b_{k}^{+}\right)}{\left(1-\delta_{N}\right) C_{k}} \\
& -\frac{\left(B-B_{j}-B_{k}\right)}{C_{k}}
\end{aligned}
$$

The Southern groups will favour disunity within a complementarity framework when their minima individual demands are low. That will incite them to accept 
the offer of the North. Acting together, their transfer demands are increased due to their strong bargaining power. Therefore, they are interested in extending the negotiation. Thus, the delay can be interpreted as the means to affirm high demands. The negotiation costs can be compensated by the anticipated higher benefit in terms of transfers. Hence, the groups characterised by low minimum demands are willing to claim more by being united.

We now focus on a situation in which the beliefs of the North lead to a separating equilibrium with one of the Southern groups and to a pooling equilibrium with the other, i.e. $p_{j}^{+}>g_{j}$ and $p_{k}^{+}<g_{k}, j \neq k$. In this situation, group $j$ (whatever its characteristics) will accept the offer $\tau^{*}\left(N, S_{j}^{+}\right)$at the first round. Its utility is written:

$$
\begin{aligned}
& U_{j}\left(\tau^{*}\left(N, S_{j}^{+}\right), 0\right)=\left[\tau^{*}\left(N, S_{j}^{+}\right)-b_{j}^{-}\right] C_{j} \text { if } S_{j} \text { is of type } b_{j}^{-} \\
& U_{j}\left(\tau^{*}\left(N, S_{j}^{+}\right), 0\right)=\left[\tau^{*}\left(N, S_{j}^{+}\right)-b_{j}^{+}\right] C_{j} \text { if } S_{j} \text { is of type } b_{j}^{+}
\end{aligned}
$$

The utility of group $k$ is:

$$
\begin{aligned}
U_{k}\left(\tau_{N k}, 0\right) & =\left[\tau_{N k}-b_{k}^{-}\right] C_{k} & & \text { if } S_{k} \text { is of type } b_{k}^{-} \\
U_{k}\left(\tau^{*}\left(S_{k}^{+}, N\right), 1\right) & =\delta_{S}\left[\tau^{*}\left(S_{k}^{+}, N\right)-b_{k}^{+}\right] C_{k} & & \text { if } S_{k} \text { is of type } b_{k}^{+}
\end{aligned}
$$

The situations $(23)+(25)$ and $(23)+(26)$ are included in the cases previously discussed. For the remaining situations, we limit ourselves to those in which the negotiation delay appears. The situation $(24)+(25)$ leads to the following relation:

$$
U_{j}\left(\tau^{*}\left(N, S_{j}^{+}\right), 0\right)+U_{k}\left(\tau_{N k}, 0\right)>U\left(\tau^{*}\left(S^{+}, N\right), 1\right)
$$

For

$$
\begin{aligned}
b_{k}^{-}< & \frac{\left(1-\delta_{N} \delta_{S}\right)\left(\delta_{S} C b^{+}-C_{j} b_{j}^{+}\right)-\left(1-\delta_{S}\right)\left(\delta_{N} \delta_{S} C b^{+}-C_{j} b_{j}^{+}-\delta_{N} \delta_{S} C_{k} b_{k}^{+}\right)}{\delta_{S}\left(1-\delta_{N} \delta_{S}\right) C_{k}} \\
& -\frac{\left(1-\delta_{N}\right)\left(B-B_{j}-B_{k}\right)}{\left(1-\delta_{N} \delta_{S}\right) C_{k}}
\end{aligned}
$$

$b_{j}^{+}$and $b_{k}^{-}$will tackle the negotiations separately. When the Southern groups are complementary and their individual demands high, they are willing to act separately. On the other hand, when they are substitutable, they will act jointly and force the negotiation delay because the beliefs of the North lead it to make a low opening proposition.

Let us now analyse the situation (24)-(26). Two cases are conceivable according to the response of the union to the initial proposition of the North:

$$
U_{j}\left(\tau^{*}\left(N, S_{j}^{+}\right), 0\right)+U_{k}\left(\tau^{*}\left(S_{k}^{+}, N\right), 1\right)>U\left(\tau^{*}\left(N, S^{+}\right), 0\right)
$$


or

$$
U_{j}\left(\tau^{*}\left(N, S_{j}^{+}\right), 0\right)+U_{k}\left(\tau^{*}\left(S_{k}^{+}, N\right), 1\right)>U\left(\tau^{*}\left(S^{+}, N\right), 1\right)
$$

These two configurations lead to the same condition, namely equation (20). Thus, although $b_{k}^{+}$bears a negotiation delay, disunity is still favoured. In the first case, the complementarity allows $b_{k}^{+}$to affirm its demands by refusing the initial offer of the North. Therefore the delay allows $b_{k}^{+}$to obtain what it expected. However, when they are substitutable, $b_{k}^{+}$is interested in joining $b_{j}^{+}$to increase its bargaining power and not to bear a delay. In the second case, the Southern groups also favour disunity instead of union. Moreover, complementarity favours $b_{j}^{+}$since it is induced to sign an agreement as of the first period, whilst, being united or acting separately, $b_{k}^{+}$is still confronted with a delay.

To resume, the different configurations analysed lead to the following results: in the presence of asymmetric information, when the Southern groups form a union (when they are substitutable) the delay favours the North. That delay corresponds to the time necessary for it to acquire the relevant information concerning the demands of the groups. On the other hand, when the Southern groups act separately (complementarity situation), the delay has several interpretations. If one of the two Southern groups is of the type $b^{+}$, while the other is of type $b^{-}$, the delay corresponds to the time necessary for $b^{+}$to dissociate from $b^{-}$. When both groups are of type $b^{+}$, the delay is used by one and/or the other group to affirm its transfer demands.

The comparison of equilibrium in complete and incomplete information in multilateral negotiations game gives the following proposition:

Proposition 6 When both groups are of type $b_{i}^{+}$, the conditions of complementaritysubstitutability in presence of asymmetric information are identical to those obtained with complete information. On the contrary, when at least one of the groups is of type $b_{i}^{-}$, the conditions of complementarity (substitution) are wider (more restrictive).

Within a multilateral negotiation with incomplete information, the existence of information asymmetry reinforces the bargaining power of complementary groups and reduces the power of substitutable groups. As with complete information, the complementarity, requiring the agreement of both groups, gives them significant bargaining power compelling the North to propose a high level of transfers. In this context, the one and/or the other Southern group will take advantage of its private information to increase its bargaining power and obtain an agreement on a high transfer level. The advantages of complementarity are therefore reinforced by the lack of information of the North. Asymmetry of information makes the conditions of complementarity obtained with complete information less restrictive. On the contrary, in the presence of substitutable groups, the North is 
interested in acquiring information on the characteristics of the groups, in order to oppose them by searching an agreement with only one of them. The possible race downwards leads the Southern groups to reveal their private information reducing their bargaining power. The drawbacks of substitutability are reinforced by informational asymmetry since, if the Southern groups request high transfers, the negotiations cannot be concluded in the first period. Thus, the conditions of substitutability are more restrictive than with complete information.

Considering these results, the South will be interested in repeating the pattern of the Kyoto Protocol. That is, the relatively rapid signing of a general agreement which makes the Southern groups complementary, followed by a discussion about the details (the Marrakech Accords in the case of the Kyoto Protocol) where the separation in two complementary groups will give them strong bargaining power to tackle the negotiations on the precise amount of the transfers.

Moreover, if both Southern groups have high demands, either complementary of substitute, they will never accept less than what they would obtain in complete information. Likewise, the North will never offer more than the maximum amount of transfers that it would have proposed with complete information. As an agreement is only reached with a transfer level corresponding to the demands of the Southern groups, the presence of asymmetry of information does not benefit them. Under these circumstances, the conditions of complementaritysubstitutability are identical with complete and incomplete information.

\section{Conclusion}

The objective of this paper has been to determine the different forms that the international agreements to enlarge the Kyoto Protocol to include the developing countries might take. The main characteristics of the negotiation are the presence of asymmetry of information about the capacities of the South to reduce their emissions and the possibility to make the negotiations last more than one round. Although we have analysed the climate change negotiations, the framework developed is wide enough to represent most of the international negotiations between the North and the South, as long as they deal with an issue where the North is willing to transfer money (or other economic advantages) in exchange of some commitment by the Southern countries; and as long as available data about the North are more accurate than data about the South.

In a bilateral framework, we have shown that asymmetry of information is not always beneficial for the developing countries coalition. Industrial countries will be interested in continuing negotiations and may delay the moment of an agreement to obtain information about the characteristics of the Southern coalition. Compared to the outcome with complete information, the developing countries will pay a negotiation cost due to the delay.

In a multilateral framework, the information asymmetry reinforces the negoti- 
ation power of the complementary coalitions, which will generally act separately, and reduces that of the substitutable coalitions, which will generally act together. Hence, the developing countries are interested in forcing a situation where they are complementary by setting, at an early stage (pre-game) of the negotiations, a high minimum level of ratifications for the agreement to come into force (as it was done for the Kyoto Protocol). If the southern coalitions manage to become complementary, they will act separately but the agreement will not be reached in the first negotiation period. The delay can now be interpreted as the time needed by the coalition with strong demands to dissociate from the coalition with weak demands. 


\section{APPENDIX}

\section{Proof of lemma 1}

See Rubinstein (1985). Nevertheless, the general principle of the demonstration is as follows. Let us consider the history for which $b^{+}$and $b^{-}$reject the offer of $N$ and suggest different offers in return. Let $\tau^{*}\left(S^{+}, N\right)$ be the offer of $b^{+}$and $\tau_{S}^{-}$the one of $b^{-}$, with:

$$
B / C \geq \tau^{*}\left(S^{+}, N\right)>\tau_{S}^{-}>\tau^{*}\left(S^{-}, N\right)
$$

After the Southern group has made its proposition, $N$ is able to determine the characteristics of its opponent.

- Behaviour of $N$ towards $b^{+}$. Faced with a proposition $\tau^{*}\left(S^{+}, N\right), N$ identifies its opponent as being $b^{+}$. Knowing that it could not propose less than $\tau^{*}\left(N, S^{+}\right)$at the next period, it will accept the counter-offer. By offering the transfer $\tau^{*}\left(N, S^{+}\right)$, its benefit is not increased compared to that which it would obtain accepting $\tau^{*}\left(S^{+}, N\right)$ a period later.

- Behaviour of $N$ towards $b^{-}$. Two cases are possible:

Observing $\tau_{S}^{-}$satisfying (31) and therefore strictly lower to $\tau^{*}\left(S^{+}, N\right), N$ knows that it is confronted with $b^{-}$. It just has to propose in the following period $\tau_{S}^{-}-\epsilon>\tau^{*}\left(S^{-}, N\right)$, with $\epsilon \rightarrow 0$ in a way that $\Pi\left(\tau_{S}^{-}, t\right)<\Pi\left(\tau_{S}^{-}-\epsilon, t+1\right)$. That strategy is profitable as it can improve its benefit. On the other hand, it deteriorates simultaneously the utility of $b^{-}$.

Suppose now that $N$ accepts $\tau_{S}^{-} . b^{-}$is then incited to deviate as it knows that $N$ will also accept $\tau^{*}\left(S^{+}, N\right)$. Since both offers $\tau_{S}^{-}$and $\tau^{*}\left(S^{+}, N\right)$ can be accepted, $b^{-}$is interested in requesting $\tau^{*}\left(S^{+}, N\right)$.

\section{Proof of lemma 2}

For each of these two cases, we consider the game at any date $t$ where the Southern group is the proposer.

- $b^{-}$makes an offer $\tau_{S}$ different from $\tau_{N}$ if the utility associated to its proposition $\tau_{S}$ is strictly higher to the one obtained with $\tau_{N}$. However, the lemma 1 stipulates that the counter-offer of $b^{-}$is equal to $\tau^{*}\left(S^{+}, N\right)$. The condition is then written:

$$
U\left(\tau_{N}, t-1\right) \leq U\left(\tau^{*}\left(S^{+}, N\right), t\right)
$$

or

$$
C\left(\tau_{N}-b^{-}\right) \delta_{S}^{t-1} \leq C\left(\tau^{*}\left(S^{+}, N\right)-b^{-}\right) \delta_{S}^{t}
$$

or

$$
\tau_{N}-\left[\left(1-\delta_{S}\right) b^{-}+b^{+} \delta_{S}\right] \leq\left(\tau^{*}\left(S^{+}, N\right)-b^{+}\right) \delta_{S}
$$


- Since $b^{+} \geq\left(1-\delta_{S}\right) b^{-}+b^{+} \delta_{S}$, we obtain: $C\left(\tau_{N}-b^{+}\right) \leq C\left(\tau^{*}\left(S^{+}, N\right)-b^{+}\right) \delta_{S}$. Thus, when $b^{-}$refuses $\tau_{N}, b^{+}$does as much.

- Suppose $b^{+}$accepts a proposition $\tau_{N}$. Hence:

$$
U\left(\tau_{N}, t-1\right) \geq U\left(\tau^{*}\left(S^{+}, N\right), t\right)
$$

or

$$
\tau_{N}-\left[b^{-} \delta_{S}+\left(1-\delta_{S}\right) b^{+}\right] \geq\left(\tau^{*}\left(S^{+}, N\right)-b^{-}\right) \delta_{S}
$$

Since $b^{-} \leq b^{-} \delta_{S}+\left(1-\delta_{S}\right) b^{+}$, we have $C\left(\tau_{N}-b^{-}\right) \geq C\left(\tau^{*}\left(S^{+}, N\right)-b^{-}\right) \delta_{S}$.

\section{Proof of lemma 3}

Suppose the Southern group does not accept $\tau_{N}$. The lemma (1) tells us that the two types of groups will suggest the same counter-offer $\tau_{S}$, with $\tau_{S} \geq b^{+}$. However, the highest proposition the Southern group can make is $\tau^{*}\left(S^{+}, N\right)$. Hence, for any transfer $\tau_{N}$ satisfying the relation $\tau_{N} \geq\left(1-\delta_{S}\right) b+\delta_{S} \tau^{*}\left(S^{+}, N\right)$, we have $\tau_{N}-b \geq\left(\tau^{*}\left(S^{+}, N\right)-b\right) \delta_{S}$. Therefore, the Southern group will accept the transfer $\tau_{N}$.

Let us specify the expression of the offer $\tau_{N}$ according to the type of the Southern group. If the group is type $b^{-}$, we have, for $b=b^{-}: \tau_{N}^{-} \geq\left(1-\delta_{S}\right) b^{-}+$ $\delta_{S} \tau^{*}\left(S^{+}, N\right)$ and for $b=b^{+}: \tau_{N}^{+} \geq\left(1-\delta_{S}\right) b^{+}+\delta_{S} \tau^{*}\left(S^{+}, N\right)=\tau^{*}\left(N, S^{+}\right)$.

Thus, although $b^{-}$is incited to behave like $b^{+}$(according to lemma 1 and 2 ), it will have more room to manoeuvre than $b^{+}$(according to lemma 3 ). I.e., its set of satisfactory propositions is wider than the one of $b^{+}$:

$$
\begin{aligned}
\tau_{N}^{-} \in A & =\left[\left(1-\delta_{S}\right) b^{-}+\delta_{S} \tau^{*}\left(S^{+}, N\right), B / C\right] \\
\tau_{N}^{+} \in B & =\left[\left(1-\delta_{S}\right) b^{+}+\delta_{S} \tau^{*}\left(S^{+}, N\right), B / C\right]
\end{aligned}
$$

and $B \subset A$. For $\delta_{S} \rightarrow 1$, we have $\tau=B / C$. The behaviour of the Southern groups $b^{-}$and $b^{+}$coincides when $b^{-}$is patient as they request both the highest transfer that $N$ can accept. Patience allows $b^{-}$to claim the requests of $b^{+}$. On the other hand, if $b^{-}$is impatient, its transfer demand becomes its reference transfer for the negotiation.

\section{Proof of lemma 4}

We successively present the behaviours of $b^{-}, b^{+}$and $N$.

- Behaviour of $b^{-}$:

$b^{-}$will accept $\tau_{N}$ if that transfer level incites it, at the next period, to renounce earnings associated to a proposition $\tau^{*}\left(S^{+}, N\right)$. It is enough that:

$$
U\left(\tau_{N}, t-1\right) \geq U\left(\tau^{*}\left(S^{+}, N\right), t\right)
$$


or

$$
\tau_{N}-b^{-} \geq\left(\tau^{*}\left(S^{+}, N\right)-b^{-}\right) \delta_{S}
$$

Due to its preference for the present, we have:

$$
\tau_{N} \geq\left(\tau^{*}\left(S^{+}, N\right)-b^{-}\right) \delta_{S}+b^{-}
$$

- Behaviour of $b^{+}$:

If $b^{+}$prefers $\tau_{S}$ to $\tau_{N}$, the following inequality has to hold:

$$
U\left(\tau_{N}, t-1\right)<U\left(\tau^{*}\left(S^{+}, N\right), t\right)
$$

or

$$
\tau_{N}<\tau^{*}\left(N, S^{+}\right)
$$

- Behaviour of two types of groups:

According to equation (32), $b^{-}$is not interested in refusing the offer $\tau_{N}$ since it will not increase its payoffs by a counter-proposition $\tau^{*}\left(S^{+}, N\right)$. On the contrary, according to $(33), b^{+}$refuses $\tau_{N}$ since it can enhance its utility by proposing $\tau^{*}\left(S^{+}, N\right)$. Thus, for an offer $\tau_{N}$ satisfying the condition (13), $b^{-}$has to accept it while $b^{+}$can refuse it to propose afterwards $\tau^{*}\left(S^{+}, N\right)$.

- Behaviour of $N$ :

If $b^{-}$accepts the offer of $\tau_{N}$ while $b^{+}$rejects it to propose afterwards $\tau^{*}\left(S^{+}, N\right)$, $N$ is able to determine the type of its opponent. In fact, it knows that for a level of transfers $\tau_{N}$, satisfying (33), $b^{-}$should not reject it (while $b^{+}$should). $N$, knowing the type of group it is negotiating with, will accept the offer $\tau^{*}\left(S^{+}, N\right)$.

\section{Proof of proposition 3}

- If $N$ opens the negotiations on an offer $\tau^{*}\left(N, S^{+}\right)$, its expected payoff is:

$$
\begin{aligned}
E \Pi^{+} & =p^{+}\left[B-\tau^{*}\left(N, S^{+}\right) C\right]+\left(1-p^{+}\right)\left[B-\tau^{*}\left(N, S^{+}\right) C\right] \\
& =B-\tau^{*}\left(N, S^{+}\right) C
\end{aligned}
$$

On the other hand, if it suggests the pure strategy $\tau_{N}$ satisfying (13), its expected payoff becomes:

$$
E \Pi^{-}=\left(1-p^{+}\right)\left[B-\tau_{N} C\right]+p^{+} \delta_{N}\left[B-\tau^{*}\left(S^{+}, N\right) C\right]
$$

According to lemma (4), the offer will be accepted by $b^{-}$and rejected by $b^{+}$. The latter will propose the transfer $\tau^{*}\left(S^{+}, N\right)$, which will be accepted by $N$ in the second period. The strategy $\tau_{N}$ is optimal for $N$ if and only if: $E \Pi^{-}>E \Pi^{+}$(condition 1 of proposition (3)). 
- If $E \Pi^{-}<E \Pi^{+}$, we are in the configuration where proposing $\tau^{*}\left(N, S^{+}\right)$ is optimal for $N$. Since that is the highest transfer that the Southern group can obtain in negotiations in complete information, it will accept it whatever its type.

- If $E \Pi^{-}=E \Pi^{+}$, the sequential equilibrium consists for $N$ in adopting the mixed strategy $\tau^{*}\left(N, S^{+}\right)$with the probability $\alpha$ and the strategy $\tau^{*}\left(N, S^{-}\right)$ with the probability $1-\alpha$ with $\alpha \in[0,1]$. $b^{+}$will accept the proposition if it is at least equal to $\tau^{*}\left(N, S^{+}\right)$while $b^{-}$will accept, in the first period, any proposition satisfying (13).

\section{Proof of proposition 4}

Since (13) is not verified, for $\tau_{N}=\tau^{*}\left(N, S^{-}\right)$, the expected payoff of $N$ is written:

$$
E \Pi^{*}=\left(1-p^{+}\right)\left[B-\tau^{*}\left(N, S^{-}\right) C\right]+p^{+} \delta_{N}\left[B-\tau^{*}\left(S^{+}, N\right) C\right]
$$

By comparing the expected payoffs associated to each strategy, we obtain the searched result

ACKNOWLEDGEMENTS: We wish to thank comments and suggestions by an anonymous referee and by the participants at the EAERE2003 conference in Bilbao (Spain) and the AFSE2003 conference in Paris (France). Alejandro Caparrós acknowledges the financial support from the European Commission (EEP Research Training Network). 


\section{REFERENCES}

Barrett, S. (1994). Self Enforcing International Environmental Agreements. Oxford Economic Papers 46: 878-894.

Barrett, S. (1997). Heterogeneous International Environmental Agreements. In C. Carraro (Ed.), International Environmental Negotiations. Strategic Policy Issues. Cheltenham: Edward Elgar.

Botteon, M. and Carraro, C. (1997). Burden-Sharing and Coalition Stability in Environmental Negotiations with Asymmetric Countries. In C. Carraro (Ed.), International Environmental Negotiations. Strategic Policy Issues. Cheltenham: Edward Elgar.

Botteon, M. and Carraro, C. (1998). Environmental Coalitions with Heterogeneous Countries: Burden-Sharing and Carbon Leakage. In A. Ulph (Ed.), Environmental Policy, International Agreements and International Trade. Oxford: Oxford University Press.

Carraro, C. and Siniscalco, D. (1993). Strategies for the International Protection of the Environment. Journal of Public Economics 2: 309-328.

Chander, P. and Tulkens, H. (1995). A Core-Theoretic for the Design of Cooperative Agreements on Transfrontier Pollution. International Tax and Public Finance 2: 279-294.

Chander, P. and Tulkens, H. (1997). The Core of an Economy With Multilateral Environmental Externalities. International Journal of Game Theory 26: 379-401.

Egteren, H. and Tang, J. (1997). Maximum Victim Benefit: A Fair Division Process in Transboundary Pollution Problems. Environmental and Resources Economics 10: 363-386.

Finus, M. (2001). Game Theory and International Environmental Cooperation. Cheltenham: Edward Elgar.

Funaki, Y. and Yamato, T. (1999). The Core of an Economy with a Common Pool Resource: A Partition Function Approach. International Journal of Game Theory 28: 157-171.

Harsanyi J., (1967-68). Games with Incomplete Information played by Bayesian Players. Management Science 14: 159-82, 320-34, 486-502.

Heal, G. (1994). Formation of International Agreements. In C. Carraro (Ed.), Trade, Innovation, Environment. Dordrecht: Kluwer Academic Press. 
Helm, C. (2001). On the existence of a cooperative solution for a coalitional game with externalities. International Journal of Game Theory 30 (1): 141-146.

Hoel, M. (1992). International Environment Convention: The Case of Uniform Reductions of Emissions. Environmental and Resource Economics 2: 141159.

Hovi, J. (2001). Decentralized enforcement, sequential bargaining, and the Clean Development Mechanism. CICERO Working Paper 2001-12.

Kreps, D. and Wilson, R. (1982). Sequential Equilibria. Econometrica 50: 863984.

Osborne, M. and Rubinstein, A. (1990). Bargaining and Market. Academic Press.

Péreau, J.-C. and Tazdaït, T. (2001). Cooperation and Unilateral Commitment in the Presence of Global Environmental Problems. Environmental and Resource Economics 20: 225-239.

Rotillon, G., Tazdaï, T. and Zeghni S. (1996). Bilateral or multilateral bargaining in the face of global environmental change? Ecological Economics 18: $177-187$.

Rubinstein, A. (1982). Perfect Equilibrium in a Bargaining Model. Econometrica 50: 97-109.

Rubinstein, A. (1985). A Bargaining Model with Incomplete Information about Time Preferences. Econometrica 53: 1151-72.

Rubinstein, A. (1988). Choice of Conjectures in a Bargaining Game with Incomplete Information. In Alvin E. Roth (Ed.), Game Theoretic Models of Bargaining. Cambridge: Cambridge University Press.

Tulkens, H. (1998). Cooperative vs. Free Riding in International Environmental Affairs: Two Approaches. In N. Hanley and H. Folmer (Eds.), Game Theory and the Environment. London: Edward Elgar. 